\title{
Carbon C 13 Phytoene
}

National Cancer Institute

\section{Source}

National Cancer Institute. Carbon C 13 Phytoene. NCI Thesaurus. Code C98833.

A 40-carbon hydrocarbon precursor of carotenoids radiolabeled to carbon C 13 and potentially used for tracer purposes of phytoene in vivo. Upon administration, phytoene is taken up and accumulates in various tissues. Upon imaging of the radioisotope, the distribution patterns and metabolism of phytoene can be further elucidated which may aid in the understanding of the bioactivity of this agent. Phytoene is a colorless precursor of many carotenoids, including the antioxidant lycopene. 